\title{
What does the local black hole mass distribution tell us about the evolution of the quasar luminosity function?
}

\author{
Luca Ciotti ${ }^{1,2,3}$, Zoltan Haiman ${ }^{3}$, and Jeremiah P. Ostriker ${ }^{4,3}$ \\ 1 Osservatorio Astronomico di Bologna, via Ranzani 1, 40127 Bologna, Italy \\ 2 Scuola Normale Superiore di Pisa, Piazza dei Cavalieri 7, 56126 Pisa, Italy \\ 3 Princeton University Observatory, Peyton Hall, 08544 NJ, USA \\ 4 Institute of Astronomy, Cambridge University, Madingley Road, CB3 0HA, \\ Cambridge, UK
}

\begin{abstract}
We present a robust method to derive the duty cycle of QSO activity based on the empirical QSO luminosity function and on the present-day linear relation between the masses of supermassive black holes and those of their spheroidal host stellar systems. It is found that the duty cycle is substantially less than unity, with characteristic values in the range $3-6 \times 10^{-3}$. Finally, we tested the expectation that the QSO luminosity evolution and the star formation history should be roughly parallel, as a consequence of the above-mentioned relation between $\mathrm{BH}$ and galaxy masses.
\end{abstract}

\section{Introduction}

The discovery of remarkable correlations between the masses of supermassive BHs hosted at the centers of galaxies and the global properties of the parent galaxies themselves [8,9,12] leads to a natural link between the cosmological evolution of QSOs and the formation history of galaxies [2]. The investigation of such interesting correlations looks promising not only to better understand how and when galaxies formed, but also to obtain information on the QSO population itself $\mathbb{1}$. Here we focus on two specific points raised by the general remarks above: 1) The use of the "Magorrian relation" to determine the QSO duty cycle at redshift $z=0 ; 2$ ) The expected relation between the cosmological evolution of the total luminosity emitted by star-forming galaxies and that of the total luminosity emitted by QSOs. As we will see, an interesting consequence of this last point is the possible existence of a physical process limiting gas accretion onto BHs at high redshifts. The observational inputs of our analysis are the Magorrian relation [12], the galaxy mass-to-light ratio (from the Fundamental Plane) [5.6], the present-day luminosity function of spheroids 14, the presentday and the integrated QSO cosmological (light) evolution [13], and finally the star formation history [11]. A possible alternative to the use of the mass-tolight ratio is the use of the Faber-Jackson [7] relation coupled with the so-called $M_{\mathrm{BH}}-\sigma$ relation [8,97. The technical details will be given elsewhere [4]. 


\section{Results}

We start our analysis by deriving the QSO's mean accretion efficiency associated with the BH's growth (see, e.g., 15]) as $\epsilon \equiv E_{\mathrm{Q}}^{\mathrm{T}} / M_{\mathrm{BH}}^{\mathrm{T}} c^{2} \simeq 0.06$, where $M_{\mathrm{BH}}^{\mathrm{T}}$ is the present-day total mass of $\mathrm{BHs}$ at the center of stellar spheroids, and $E_{\mathrm{Q}}^{\mathrm{T}}$ is the total energy emitted by all QSOs over the entire life of the Universe. We then derive the QSO (mean) duty cycle $f_{\mathrm{Q}}$ in two ways:

$$
<f_{\mathrm{Q}}>_{x} \equiv \frac{\epsilon c^{2} M_{\mathrm{BH}}(x)}{\int_{0}^{\infty} L_{\mathrm{Q}}(x, t) d t}, \quad<f_{\mathrm{Q}}>_{x} \equiv \frac{N_{\mathrm{BH}}^{>}\left[M_{\mathrm{BH}}(x)\right]}{N_{\mathrm{Q}}^{>}\left[L_{\mathrm{Q}}(x, 0)\right]},
$$

where $L_{\mathrm{Q}}(x, t)$ is such that QSOs brighter than $L_{\mathrm{Q}}(x, t)$ (whose number is $\left.N_{\mathrm{Q}}^{>}\left[L_{\mathrm{Q}}(x, t)\right]\right)$ emit the fraction $x$ of $L_{\mathrm{Q}}^{\mathrm{T}}(t)$, and $M_{\mathrm{BH}}(x)$ is such that all BHs heavier than $M_{\mathrm{BH}}(x)$ (whose number is $N_{\mathrm{BH}}^{>}\left[M_{\mathrm{BH}}(x)\right]$ ) sum up to $x M_{\mathrm{BH}}^{\mathrm{T}}$ (at $\mathrm{z}=0$ ). We found that the two ways give consistently $<f_{\mathrm{Q}}>_{0.1} \simeq 0.003$ and $<f_{\mathrm{Q}}>_{0.9} \simeq 0.006$, in good agreement with theoretical results [1]. Our result is similar in spirit but different in detail from that derived in 10 , who obtained a $Q S O$ lifetime of $\simeq 10^{7} \mathrm{yr}$ at a Hubble epoch of $\simeq 10^{9} \mathrm{yr}$ or, in our terms, $f_{\mathrm{Q}} \simeq 10^{-2}$. Finally, an interesting expectation based on the Magorrian relation would be a parallel evolution of the QSO accretion and star formation histories. As is well known, at $z \lesssim 2$ the QSO total luminosity and the UV luminosity associated to the star formation history are indeed roughly parallel but at $z \gtrsim 2$ they have clearly divergent slopes. A fit shows that the QSO luminosity evolution is reasonably well fitted under the assumptions that $\mathrm{BHs}$ are accreting at one tenth of the Eddington luminosity, as computed under the standard assumption of pure electron scattering. A global picture could then be that at low redshift the BH accretion (and star formation) are limited by the available amount of gas, while at high redshift some extra source of opacity (as for example bremsstrahlung opacity [3]), able to reduce the Eddington luminosity of one order of magnitude (with respect to the pure electron scattering case), is at work [4].

\section{References}

1. L. Ciotti, J.P. Ostriker: ApJ, 551, 131 (2001)

2. L. Ciotti, T.S. van Albada: ApJ, 552, L13 (2001)

3. L. Ciotti, B. Draine, J.P. Ostriker: in preparation

4. L. Ciotti, Z. Haiman, J.P. Ostriker: in preparation

5. G. Djorgovski, M. Davis: ApJ, 313, 59 (1987)

6. A. Dressler et al.: ApJ, 313, 42 (1987)

7. S.M. Faber, R.E. Jackson: ApJ, 204, 668 (1976)

8. L. Ferrarese, D. Merritt: ApJ, 539, L9 (2000)

9. K. Gebhardt, K., et al.: ApJ, 539, L13 (2000)

10. M.G. Haehnelt, P. Natarajan, M.J. Rees: MNRAS 300, 827 (1998)

11. P. Madau, L. Pozzetti: MNRAS, 312, 9 (2000)

12. J. Magorrian, et al.: AJ, 115, 2285 (1998)

13. Y.C. Pei: ApJ, 438, 623 (1995)

14. P. Salucci et al.: MNRAS, 307, 637 (1999)

15. A. Soltan: MNRAS, 200, 115 (1982) 